The University of Southern Mississippi

The Aquila Digital Community

Faculty Publications

6-10-2006

\title{
Energy Storage In Cold Non-Elastic Deformation of Glassy Polymers
}

\author{
E.F. Oleinik \\ Russian Academy of Sciences \\ S.N. Rudnev \\ Russian Academy of Sciences \\ O.B. Salamatina \\ Russian Academy of Sciences \\ S.V. Shenogin \\ Rensselaer Polytechnic Institute \\ M.I. Kotelyanskii \\ Rudolph Technologies, Inc., mkotelyanskii@rudolphtech.com
}

See next page for additional authors

Follow this and additional works at: https://aquila.usm.edu/fac_pubs

Part of the Chemistry Commons

\section{Recommended Citation}

Oleinik, E., Rudnev, S., Salamatina, O., Shenogin, S., Kotelyanskii, M., Paramzina, T., Nazarenko, S. (2006). Energy Storage In Cold Non-Elastic Deformation of Glassy Polymers. E-Polymers.

Available at: https://aquila.usm.edu/fac_pubs/2341

This Article is brought to you for free and open access by The Aquila Digital Community. It has been accepted for inclusion in Faculty Publications by an authorized administrator of The Aquila Digital Community. For more information, please contact Joshua.Cromwell@usm.edu. 


\section{Authors}

E.F. Oleinik, S.N. Rudnev, O.B. Salamatina, S.V. Shenogin, M.I. Kotelyanskii, T.V. Paramzina, and S.I. Nazarenko 


\title{
Energy Storage in Cold Non-elastic Deformation of Glassy Polymers
}

\author{
E.F.Oleinik, ${ }^{1}$ S.N.Rudnev, ${ }^{1}$ O.B.Salamatina, ${ }^{1}$ S.V.Shenogin, ${ }^{2}$ M.I.Kotelyanskii, ${ }^{3}$ T.V. \\ Paramzina, $^{1}$ S.I.Nazarenko ${ }^{4}$
}

${ }^{1}$ Semenov Institute of Chemical Physics Russian Academy of Sciences, Kosygin Str. 4, Moscow, 119991 Russia. Fax: 7-095-137-8284. Email: efoleinik@center.chph. ras.ru]

${ }^{2}$ Rensellar

${ }^{3}$ Numerical Dpt. Rudolph Technologies, Inc. Flanders, N.J 07836. U.S.A. Fax: 973.448.4404. Email: mkotelyanskii@rudolphtech.com

${ }^{4}$ School of Polymers and High Performance Materials, Univ.of Southern Mississipi, Hattiesburg MS 39406-0001, USA. Fax: 601-266-5504. Email: sergei.Nazarenko@ usm.edu]

(Received: 24 August, 2005; published: 10 June, 2006)

\begin{abstract}
Experimental results on work $\mathrm{W}(\varepsilon)$, heat $\mathrm{Q}(\varepsilon)$ and stored energy $\mathrm{U}(\varepsilon)$ of deformation for glassy polymers such as linear PS, PC, PMMA, Polyimid, amorphous PET, thermotropic aromatic polyesters, Vectra ${ }^{\mathrm{TM}}$ for example, crosslinked epoxy are presented. All the data was obtained by a deformation calorimetry technique. Loading and unloading of samples were performed at room temperature with strain rate $\dot{\varepsilon}=10^{-2}-10^{-4} \mathrm{sec}^{-1}$ under uniaxial compression up to engineering strains of $\varepsilon_{\text {def }}=40-50 \%$. During straining all polymers accumulate an excess of the latent energy $U(\varepsilon)$. Elastic fraction of the energy is released completely at sample unloading and only residual $\cup_{\text {res }}(\varepsilon)$ energy is conserved in samples. The latent energy $\cup_{\text {res }}(\varepsilon)$ grows up to $\varepsilon_{\text {def }}=20-25 \%$ and levels off then. Shapes of the $U_{\text {res }}(\varepsilon)$ curves are the same (S-shape) for all polymers. However, the saturation level is different for each polymer. The ratio $U(\varepsilon) / W(\varepsilon)$ was also measured. It was found that at strains $\varepsilon_{\text {def }}<\varepsilon_{y}\left(\varepsilon_{y}\right.$ - strain at the yield point) $U(\varepsilon) / W(\varepsilon) \approx 100 \%$. l.e. all $W$ is stored by sample in a form of $U$. The ratio decreases up to $60-30 \%$ for different polymers at higher strains. Release of the residual energy $U_{\text {res }}$ (DSC measurements) and strain $\varepsilon_{\text {res }}$ (thermally stimulated strain recovery technique) was measured for deformed and unloaded samples at heating. It was found that about $85-90 \%$ of $U_{\text {res }}$ stored by samples is released in glassy state of polymers (below $T_{g}$ ). The $U_{\text {res }}$ is related to a small fraction of $\varepsilon_{\text {res }}$, only to $7-10 \%$. The rest of $U_{\text {res }}$ and $\varepsilon_{\text {res }}$ are recovered at the softening (devitrification) interval, around $\mathrm{T}_{\mathrm{g}}$. Computer modeling (molecular dynamics) of an isothermal shear deformation was performed for 2-dimentional two component atomic glass containing 500 Lennard-Jones particles of two different diameters. It was found that localized deformation events are of anelastic nature. The $\varepsilon_{\text {an }}$ appears at early deformation stage in a form of localized shear events (transformations). Such events are nucleated in a sample and merged and united at later deformation stages, when concentration of the events becomes high enough. Finally, merged transformations form kind of shear band crossing entire sample. On the basis of experimental data and computer modeling the deformation mechanism for glassy polymers is proposed. The first stage of the process is the nucleation of "the carriers of non-elastic strain", anelastic shear transformations (ASTs). All these ASTs are energetically excited. The concentration of the ASTs is responsible for the
\end{abstract}

\footnotetext{
* The paper was presented as the Invited Lecture at the VI International Polymer Seminar in Gliwice, Poland, June 2005.
} 
amount of $U_{\text {res }}(\varepsilon)$ stored by a sample. It is suggested that such nucleation is the rate-controlling step in non-elastic deformation of any non-covalent glass. Saturation of the stored energy is defined by the reaching the steady state regime in carrier's concentration. In this regime the rates of nucleation and termination (decrease of the stored local energy by AST) of carriers becomes equal. The termination proceeds spontaneously and easy (fast). The decrease of local energy of ASTs follows by local uncoiling of chains and by an appearance of new, extended chain conformers. However, such uncoiling is not the rate-controlling step for entire deformation process. Suggested mechanism very well describes all existing experimental facts. Deformation mechanisms for glasses seriously differ from that operating in rubbers and crystals.

\section{Introduction}

Plastic deformation of glassy polymers is a topic of the intensive study now [1-6]. Two reasons, at least, are responsible for that. One is related to fundamental science. A reasonable picture of deformation processes active in polymer crystals exists [7-9]. Plastic deformation in them obeys general classic rules of crystallographic plasticity. Long chain molecules only decrease the number of glide systems potentially active from the crystal symmetry point of view $[8,9]$.

However, the understandings of deformation mechanisms operating in glassy polymers do not reach a desirable level of clarity yet. This is true for any glassy solids. Different physical pictures of the process had been proposed for glassy polymers [2, 4, 6, 10-14]. However, choice between them is not easy and definite yet. Understanding of deformation mechanisms acting in glassy matter is an important goal of solid state physics.

The second reason is the fast growth of a technical interest in polymer materials. Structural materials, glassy and semi-crystalline polymers and composites with polymer matrices have to show high level of mechanical performance. Therefore, study of their mechanical behavior including deformation mechanisms is an important technical and material science task.

Mechanical work $W$ is expended to change the shape and size of a body, i.e. to deform it. In a deformation process in a solid the work $W$ is transformed in two ways, either production of new structural defects or is dissipated into deformation heat $Q$. For glassy matter the term "structural defect" is not well defined [1]. In this paper under the term "new defect" we mean the change of initial local structure of some site of a glass into new configuration with new atomic coordinates. Atomic displacements in such defect give local strain $\varepsilon_{\text {loc. }}$. Appearance of structural defects always increases the internal energy $U$ of a deformed material [1].

If part of $U$ is conserved by a sample after its complete unloading, this part of $U$ we will call $U_{\text {res }}$, the residual or stored energy of deformation. The difference $\Delta U=\left(U-U_{\text {res }}\right.$ ) is the energy changes relating to a linear-elastic (Hookian in tension) fraction of a total $U$. The $\Delta U$ disappears from a solid sample fast and completely at unloading. So, the deformed and unloaded sample from the point of view of its energy contains only the stored energy of cold work $U_{\text {res }}$ the amount of which is defined by concentration of new deformation defects produced by an external force during a deformation process.

The necessary condition for a deformed and unloaded sample to accumulate $U_{\text {res }}$ is the existence of a non-zero level of residual strain, $\varepsilon_{\text {res }}>0$ at deformation temperature $\mathrm{T}_{\text {def }}$ (isothermal straining). The condition implies that the $U_{\text {res }}$ may exist only in the solid materials because only solids, but not liquids, may carry $\varepsilon_{\text {res }}$ after 
unloading. The $U_{\text {res }}$ is stored by two components of the $\varepsilon_{\text {res }}$, by anelastic $\varepsilon_{\text {an }}$ and plastic $\varepsilon_{\mathrm{pl}}$ strain components, i.e. $\varepsilon_{\mathrm{res}}=\varepsilon_{\mathrm{pl}}+\varepsilon_{\mathrm{an}}$ and $\varepsilon_{\mathrm{def}}=\varepsilon_{\mathrm{res}}+\varepsilon_{\mathrm{le}}$, where $\varepsilon_{\mathrm{le}}$ is the linear-elastic component.

Due to motion of atomic or molecular elements of a sample structure involved into a deformation process a generalized "friction" appears and deformation heat is produced. Usually, at moderate strains the magnitude of the linear-elastic heat $Q_{l e}$ in polymer glasses is quite small in comparison with a heat of non-elastic and plastic deformation, $Q_{n e}$ and $Q_{p l}$ correspondingly. The $Q_{l e}$ disappears from a sample after it's unloading and $Q_{\text {res }}$ is the deformation heat related to $\varepsilon_{\text {res. }}$. $Q_{\text {res }}=Q_{n e}-Q_{l e}$ and $Q_{n e}=$ $Q_{\mathrm{an}}+\mathrm{Q}_{\mathrm{pl}}$.

Measured magnitudes of $\mathrm{W}(\varepsilon), \mathrm{Q}(\varepsilon)$, and $\mathrm{U}(\varepsilon) / \mathrm{W}(\varepsilon)$ or $\mathrm{Q}(\varepsilon) / \mathrm{W}(\varepsilon)$ ratios also give information about deformation mechanisms acting in solids. The values $\mathrm{U}(\varepsilon) / \mathrm{W}(\varepsilon)$ or $\mathrm{Q}(\varepsilon) / \mathrm{W}(\varepsilon)$ show which part of the expended work $\mathrm{W}(\varepsilon)$ is transforming into the $\mathrm{Q}(\varepsilon)$ or $\mathrm{U}(\varepsilon)$ in a deformation process.

Measurement of $W(\varepsilon), Q(\varepsilon)$ and $U(\varepsilon)$ is possible at loading and unloading, and the latter regime provides information about relaxations existing in deformed materials. The loading and unloading conditions and the structure of a sample define the proportion of U/W or Q/W at any strain and measurements of the U/W or Q/W as a function of strain is a common practice in deformation thermodynamic experiments [15-18, 22-25]. For example, crystalline metals convert nearly all expended W into deformation heat $Q$ [15] but for glassy polymers large fraction of $W$ is converted into $\bigcup_{\text {res }}[4,16-18]$. The amount of $U_{\text {res }}$ depends on residual strain $\varepsilon_{\text {res }}$ accumulated by a body.

Experiments had shown [15] that the energy storage appears in solids only at cold deformation. Critical level of $\mathrm{T}_{\text {def }}$ exists for any solid. If straining is performed under isothermal conditions at $T_{\text {def }}>T_{\text {crit }}$ the energy $U_{\text {res }}$ does not remain in a sample, i.e. $U_{\text {res }}=0$ for such deformation conditions. Table 1 demonstrates it for solids of different structure. The Table shows again that the energy storage phenomenon may exist only in cold worked solids. The solidity of a body and not its symmetry, is the necessary condition for a $U_{\text {res }}$ storage. Such situation explains the reason why we consider in this paper only the cold non-elastic deformation (cold work). Experiments had also shown that amount of $U(\varepsilon)$ and $U_{\text {res }}(\varepsilon)$ increases with $T_{\text {def }}$ decrease. This was found for both, crystalline metals [15] and glassy polymers [4].

Deformed state of a solid is an excited one and therefore it tends to relax isothermally in time after unloading. The relaxation time may be very large if the relaxation temperature is far below $\mathrm{T}_{\mathrm{g}}$ (or $\mathrm{T}_{\text {recr }}$ for crystals). The relaxing in time strain component in a solid material is called $\varepsilon_{a n}$, (Fig. 1). $\bigcup_{\text {res }}$ relaxes together with $\varepsilon_{\text {an }}$. The relaxation of $U_{\text {res }}$ and $\varepsilon_{\text {res }}$ or $\varepsilon_{\text {an }}$ becomes faster at a deformed sample heating. When $T_{g}$ or $T_{\text {recr }}\left(a\right.$ little above $T_{g}$ ) are reached at heating all stored $U_{\text {res }}$ and $\varepsilon_{\text {res }}$ disappeares completely and the normal (non-deformed) structure of a solid is recovered. However, there are examples of non-complete recovery of $\varepsilon_{\text {res }}$ at such heating of glassy and semi-crystalline polymers [4, 16]. Such component of strain is called visco-plastic, $\varepsilon_{\mathrm{vp}}$ (Fig.1).

The stored $U_{\text {res }}$ may be erased from a deformed sample partially. Heating of the glassy sample containing $U_{\text {res }}$ and $\varepsilon_{\text {res }}$ up to $T<T_{g}$ creates such a situation. Such heating procedure is called sometimes the "partial heating". The heating provides many possibilities to trace amount of $U_{\text {res }}$ stored by different strain components 
coexisting in a deformed and unloaded glass [4, 17, 18]. Reloading of a sample after the partial heating permits to investigate the reaction of glass with the intermediate structure to the second and next loadings.

Tab. 1. Energy storage in a cold and hot deformation of solids

\begin{tabular}{|c|c|}
\hline GLASSES & CRYSTALS \\
$\begin{array}{c}\text { Hot deformation } \\
\mathrm{T}_{\text {def }} \geq \mathrm{T}_{\mathrm{g}}\end{array}$ & $\begin{array}{c}\text { Hot deformation } \\
\mathrm{T}_{\text {def }} \geq \mathrm{T}_{\text {recr }}<\mathrm{T}_{\mathrm{m}} \\
\text { Energy storage - No } \\
\text { Energy storage - No }\end{array}$ \\
\hline $\begin{array}{c}\text { Cold deformation } \\
\mathrm{T}_{\text {def }}<\mathrm{T}_{\mathrm{g}} \\
\text { Energy storage - Yes }\end{array}$ & $\begin{array}{c}\text { Cold deformation } \\
\mathrm{T}_{\text {def }}<\mathrm{T}_{\text {recr }} \\
\text { Energy storage -Yes }\end{array}$ \\
\hline
\end{tabular}

Temperatures of: deformation $T_{\text {def }}$, recrystallisation $T_{\text {recr }}$, melting $T_{m}$, glass transition $T_{g}$.

Fig. 1 demonstrates macroscopic strain components coexisting in a solid and their recovery after unloading.

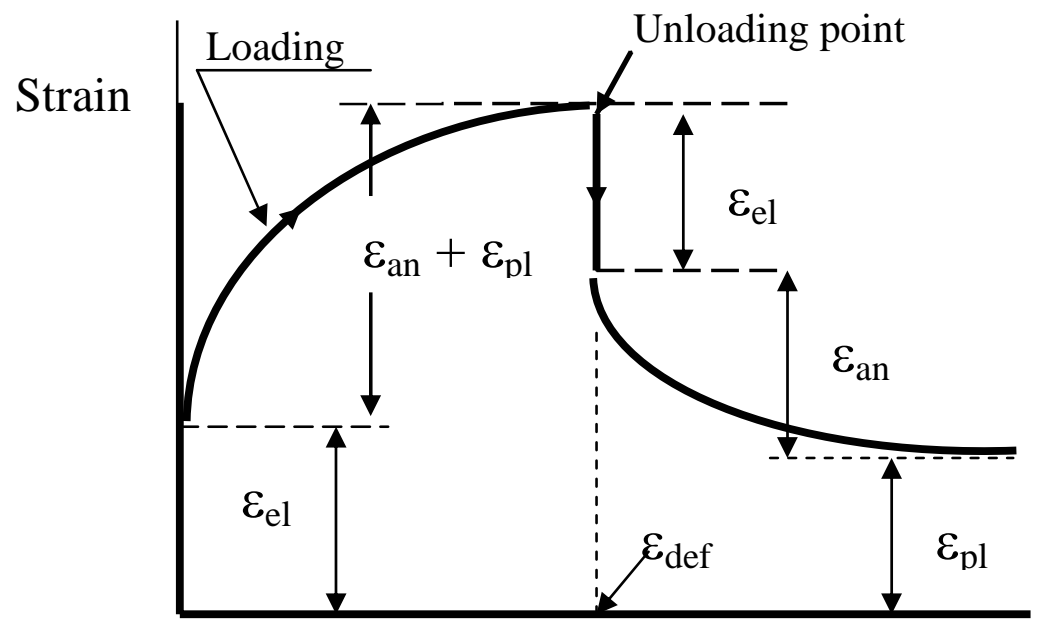

Time

Fig. 1. Components of total strain $\varepsilon_{\text {def }}$ accumulated by a solid sample at it's loading by a constant stress and the disappearance of the components after unloading. $T_{\text {def }}=$ const. The following relations are valid: $\varepsilon_{\mathrm{el}}=\varepsilon_{\mathrm{le}}+\varepsilon_{\mathrm{nle}}$, where $\varepsilon_{\mathrm{le}}$ is linear-elastic and $\varepsilon_{\text {nle }}-$ nonlinear-elastic components; $\varepsilon_{\mathrm{pl}}$-plastic component; $\varepsilon_{\mathrm{pl}}=\varepsilon_{\text {res }}$ apparent $=\varepsilon_{\text {conf }}+$ $\varepsilon_{\mathrm{vp}}$ below $\mathrm{T}_{\mathrm{g}}$. In polymers $\varepsilon_{\mathrm{conf}}$ is conformational and $\varepsilon_{\mathrm{vp}}$ is visco-plastic components. $\varepsilon_{\text {an }}$ is anelastic (delayed elastic) component [1]. $\varepsilon_{\text {an }}$ exists only in the solid state of matter, i.e. below $T_{g}$ or $T_{m}$. $\varepsilon_{\mathrm{pl}}$ is the component able to recover only in the liquid (rubbery) state of matter, i.e. at $T \geq T_{g}\left(T_{m}\right)$.

Energy storage in cold plastic deformation was extensively studied for large number of crystalline metals. Last comprehensive review was published in 1973 [15]. Several earlier good reviews also exists [20-25]. Many glassy polymers were studied [4, 17, 18, 26-28]. However, limited amount of the deformation thermodynamic data exists for glassy metals and polymer crystals. 
Study of energy and heat of deformation was performed for polymers in the rubbery state [29, 30]. Prof. Y.Godovskyi had introduced the main facts and their interpretation to the field. Unfortunately, he passed away recently [31]. It should be mentioned here that polymers in the rubbery state are not able to perform the energy storage in the given definition of the term, because rubbers are not solids. However, measurements of heat and energy evolution in the thermoelastic deformation of rubbers gave new, very useful information about structural and conformational reorganizations in them under loading and unloading [29, 30].

Thermodynamic data on deformation processes provide the bases for comparison of mechanical behavior for materials with completely different organization, spatially ordered or disordered, low and high molecular weight, for cured or linear polymers and rubbers.

Undoubtedly, the mechanisms providing structural alterations in solids of different organization under deformation are different. However, comparisons of the thermodynamic quantities characterizing deformation processes in different materials are useful and informative because these quantities reflect $W$ and $U$ transformations in given material. It was shown in the number of publications [4, 15-18, 20, 21, 26-30, 32] and it will be shown in this paper again.

In this paper we will try to answer several questions:

- What is the behavior of the stored energy of cold work $\left(U_{\text {res }}\right)$ at straining of glassy polymers?

- What kind of structural transformations in glassy polymers are responsible for the storage of the energy of cold work and for the deformation heat $Q$ production?

- What are the fractions of U/W or Q/ W for conventional glassy polymers and how the fractions are different in polymer glasses and other materials, crystalline metals for example? And what are the reasons for the differences?

- Can new physical ideas and new deformation mechanisms be extracted from the deformation thermodynamic measurements on polymer glasses?

\section{Studied polymers and experimental conditions of measurements}

In this paper we shall consider isothermal deformation behavior of different glassy polymers, such as:

-linear flexible chain polymers: polystyrene (PS), polymethylmethacrylate (PMMA), polyimid PI [(poly-benzo phenon)-imid], amorphous polyethylenetherephtalate (amPET);

-semi-rigid chain polymers: polycarbonate (PC) and thermotropic aromatic polyester Vectra ${ }^{\mathrm{TM}}$;

-chemically cross-linked polymers - epoxy-aromatic amine networks (EAAN) of different degree of cross-linking. EAAN was prepared by cure reaction of diglycidil ether of resorcinol with aromatic amines [33].

All mentioned polymer glasses except epoxy networks were of commercial origin with sufficiently high molecular masses. No traces of crystallinity (X-ray, DSC) were found in the most of the studied samples. Only am-PET had shown appearance of low concentration of crystallites after deformation (deformation induced crystallization) and Vectra ${ }^{\mathrm{TM}}$ had shown crystallinity about 5-10\% [26]. For mechanical measurements we machined samples (cylinders, $D=2-3$ and $L=3-4 \mathrm{~mm}$ ) from polymer bulk. Measurements of $\mathrm{W}$ and $\mathrm{Q}$ were performed always at room temperature in the uniaxial compression mode of loading with rates $\varepsilon=10^{-2}-10^{-4} \mathrm{sec}^{-1}$. Compression avoids samples from brittle fracture, crazing and neck formation. Unloading was performed at the same temperature and strain rate as the loading. 


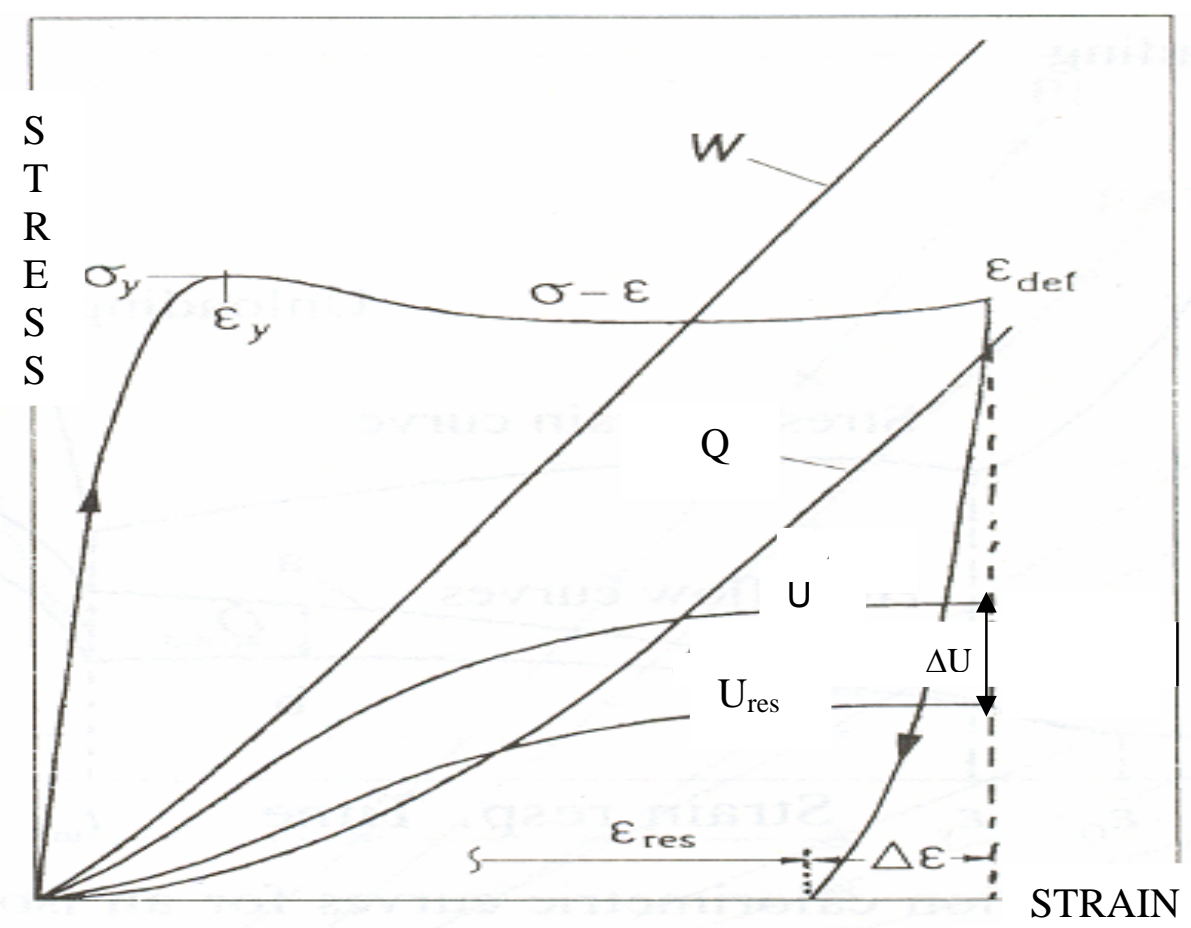

Fig. 2. Variations of $\mathrm{W}(\varepsilon), \mathrm{Q}(\varepsilon), \mathrm{U}_{(\varepsilon)}, \mathrm{U}_{\text {res }}(\varepsilon)$ and $\Delta \mathrm{U}_{(\varepsilon)}$ at loading and unloading of glassy polymers (see text) [38]. $\tilde{\Delta \varepsilon a m o u n t ~ o f ~ s t r a i n ~ r e l a x e d ~ d u r i n g ~ s a m p l e ~ u n l o a d i n g ~}$ at $T_{\text {unl }} \sigma_{\mathrm{y}}$ and $\varepsilon_{\psi}$-yield stress and strain. $\varepsilon_{\text {res }}-$ residual strain, $\Delta \varepsilon=\varepsilon_{\text {an }}+\varepsilon_{\mathrm{pl}}+\varepsilon_{\mathrm{vp}}$ (see Fig.1). $\Delta \mathrm{U}$ - elastic energy collected by sample.

Deformation temperature was always far below $T_{g}$ of studied polymers. The smallest difference $\left(T_{g}-T_{\text {def }}\right)$ was for am-PET $\left(T_{g} \approx 70^{\circ} \mathrm{C}\right)$ and the largest for $P I\left(T_{g} \approx 340^{\circ} \mathrm{C}\right)$. Deformation was homogeneous up to strains $\approx 10 \%$ and macroscopic shear bands have usually appeared after that strain. Deformation calorimeter (DC), the machine (home made) permitting to measure simultaneously the deformation work W (area under stress-strain curve) and deformation heat $\mathrm{Q}$. More detailed description of the used $D C$ is given in $[4,16-19]$.

Using the First Law one can calculate the energy change of a sample,

$$
\Delta \mathrm{U}=\mathrm{Q}+\mathrm{W}
$$

for any strains. Fig.2 shows typical curves for glassy polymers of changes of W, $Q$ and $U$ with $\varepsilon_{\text {def }}$ during glassy sample straining and unloading.

The elastic fraction of energy $\Delta U=\left(U-U_{\text {res }}\right)$ is usually smaller than $U_{\text {res }}$ for all glassy polymers at room temperature loading and reaches typically $\approx 20-25 \%$ of $\mathrm{U}_{\text {res }}$ at $\varepsilon_{\text {def }}=$ $30-50 \%$. The $\Delta \mathrm{U}$ disappears fast at unloading.

We recorded also DSC traces for deformed samples and have measured the thermally stimulated recovery (TSR) of the stored energy $U_{\text {res }}$ and residual strain $\varepsilon_{\text {res. }}$. In such measurements deformed samples were heated a little above $T_{g}$, just up to the high temperature boundary of the glass transition temperature interval. In several cases $\mathrm{T}_{\text {def }}$ was low, up to $-170^{\circ} \mathrm{C}$. Measurements at low temperatures were done not by DC, but with the help of DSC and TSR. Experimental procedures for the measurements can be found in literature [4, 16-19].

Our interest in this work was limited to the small and moderate strains, up to $\varepsilon_{\text {def }}=40$ $50 \%$. In this strain range initiation of plastic carriers, the local deformation defects carrying some amount of local strain, and the first stages of their after-nucleation 
propagation (development) happens. In other words here we will touch mostly mechanisms of the non-elastic deformation onset and initial stages of steady plastic flow.

\section{The energy storage}

Data on stored energy $U_{\text {res }}$ for different glassy polymers are shown in Fig.3. The $\cup_{\text {res }}\left(\varepsilon_{\text {def }}\right)$ curves are qualitatively similar for all studied materials. $U_{\text {res }}$ grows systematically up to strains of about $\varepsilon_{\text {def }} \approx 25-35 \%$ and becomes saturated then. The saturation level depends on polymer nature.

The growth of $U_{\text {res }}(\varepsilon)$ up to $25-30 \%$ strain manifests an increase in the concentration of deformation defects in polymer produced during loading. Clearly each defect carries some local $U^{\text {loc }}$ and non-elastic local strain $\varepsilon^{\text {loc }}$. Saturation of $U_{\text {res }}=f\left(\varepsilon_{\text {def }}\right)$ curves at $\varepsilon_{\text {def }} \approx 25-30 \%$ manifests the transition of the deformation process into the energetically steady state conditions. The strain level where the saturation starts is approximately the same for all the studied polymers. The steady-state process may appear only when the rate of the deformation defect's production becomes equal to the rate of their termination, the loss of an excitation (partially or completely) by them. It should be mentioned that not only $U_{\text {res }}(\varepsilon)$ come to the steady state regime at plastic flow. Measurements with PMMA had shown that deformation process becomes steady mechanically also [34].

Different levels of $U_{\text {res }}$ saturation for different polymers at saturation may indicate the following two situations: (1) concentrations of deformation defects are different in different polymers, or (2) the defect concentrations are the same (close) in polymers with different chemistry, but the energy excess stored in any individual defect with the same $\varepsilon^{\text {loc }}$ is different for different polymers. The first situation looks more appropriate now because the modules and yield stresses are close for organic glasses at room temperature [35]. However, there is not enough data yet permitting to discriminate these two possibilities.

Magnitudes of $U_{\text {res }}$ at the saturation level are comparatively small. They are only fractions of melting enthalpies of polymer crystals (for example $\approx 296 \mathrm{~kJ} / \mathrm{kg}$ for PE and about $150 \mathrm{~kJ} / \mathrm{kg}$ for iso-PP). Such low level of $U_{\text {res }}$ stored by deformed polymer glasses are related to small values of $W$ necessary for creation of macroscopic plastic flow at room temperature deformation of such materials. Plastic flow starts at room temperature in the most of the polymer glasses at strains $\varepsilon_{\text {def }}=5-12 \%$. Amount of $W$ necessary to deform glassy polymer to such strains is about $5-10 \mathrm{~kJ} / \mathrm{kg}(\mathrm{PI}$, EAN, PS) [4]. Such small values of $W$ reflect the fact that organic polymer glasses are comparatively weak against external mechanical forces. It is expectable because the Van-der-Waals intermolecular interactions responsible for solidity of organic polymers are weak. Even small concentration of deformation defects is sufficient to provide macroscopic yield and plastic flow in the materials. The low amounts of $\mathrm{W}$ and $U$ necessary for creation of plastic flow shows that only small fraction of atoms and atomic groups of the solids are responsible for resistance of the materials to plastic deformation. Only small number of the interatomic/intermolecular contacts should be violated to involve an organic solid into the global plastic flow. The picture seriously contradicts the melting of polymer crystals where all intermolecular contacts should be disturbed by thermal energy. 


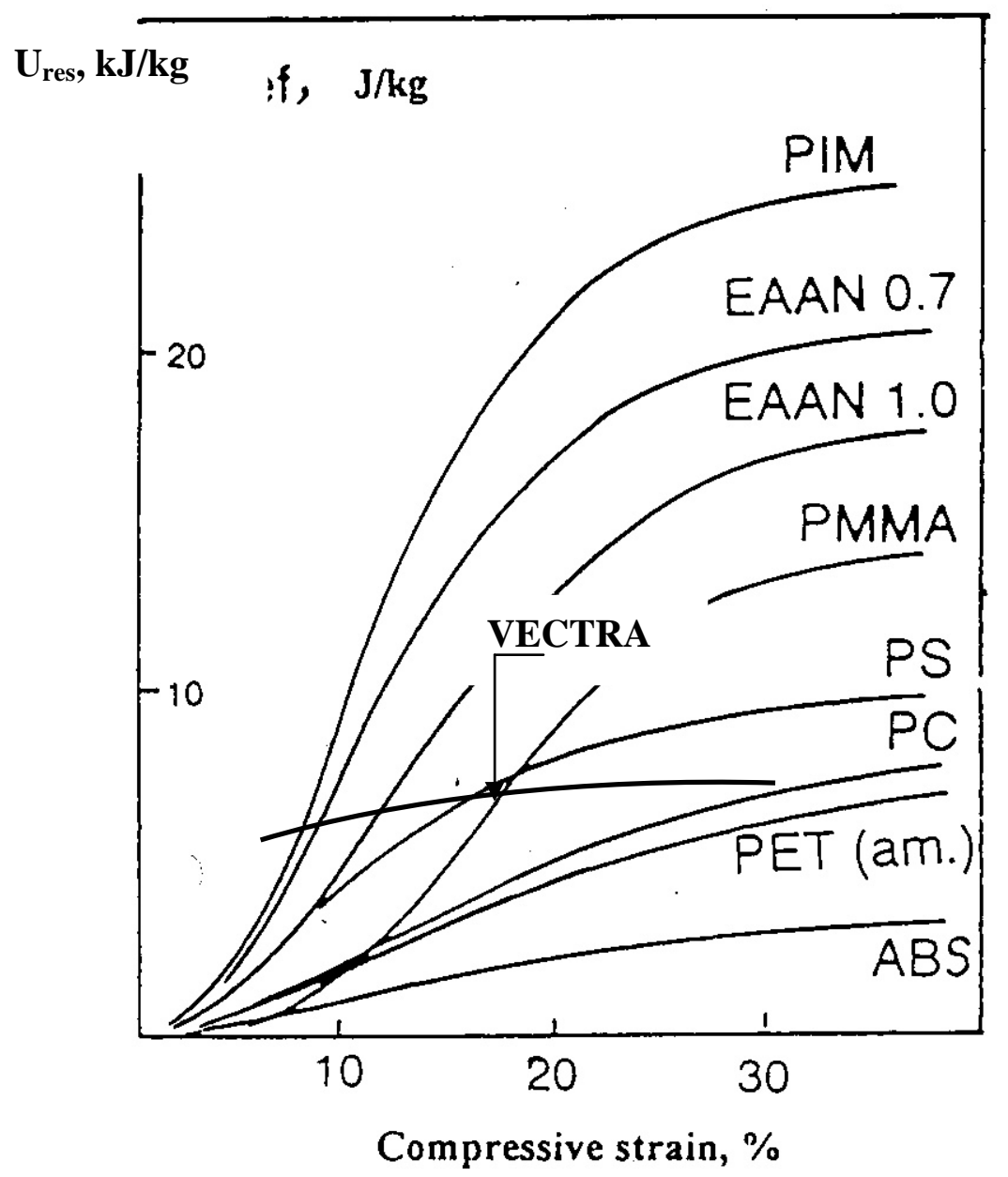

Fig.3. Accumulation of $U_{\text {res }}\left(\varepsilon_{\text {def }}\right)$ in room temperature compressive loading of different glassy polymers $[4,16,26]$.

Accumulation of $U_{\text {res }}(\varepsilon)$ shown in Fig. 3 enforce one to draw several important conclusions.

- There is the maximal amount of $U_{\text {res }}(\max )$ that a given polymer is able to accumulate at constant temperature deformation. The $U_{\text {res }}(\max )$ is reached typically at the beginning of saturation (Fig. 3) and levels off then.

- For each individual polymer the level of the stored energy $U_{\text {res }}(\max )$ at any strain increases when $T_{\text {def }}$ is decreased [4]. However, it does not look that the values of $U_{\text {res }}(\max )$ simply correlates with the $\Delta T=\left(T_{g}-T_{\text {def }}\right)$. For example, $P S$ and PMMA or $P C$ and EAAN-1.0 have close $T_{g}$ and $\Delta T$. However, they show different $U_{\text {res }}(\max )$.

- Amount of accumulated $U_{\text {res }}$ does not correlate with a flexibility of macromolecules. Polymers with the comparatively rigid chains, "Vectra" for example, store lower amount of $U_{\text {res }}$ in comparison with polymers consisting of more flexible chains. It was found that amount of $\mathrm{W}$ expended for comparatively rigid chain polymers (aromatic polyesters) is usually smaller than for flexible-chain polymers [4, 16-17, 26-28].

- Linear and chemically cross-linked polymers (EAAN) store about the same amount of $U_{\text {res }}(\varepsilon)$ for all strains. It demonstrates that chemical cross-links do not introduce serious structural obstacles for yield and plastic flow. Probably such behavior of the cross-linked polymers manifests a process where the elementary plastic transformations are smaller in size in comparison with intercross-link distances [33].

- No jumps in $U_{\text {res }}(\varepsilon)$ accumulation were detected in the yield tooth strain range. It shows that the beginning of macroscopic yield is not connected with any structural or 
thermodynamic transitions in the materials. It suggests that yield tooth in glassy polymers is a kinetic phenomenon, the same as in crystals.

Important information about a deformation process and its development gives the ratios $\mathrm{U} / \mathrm{W}$ or $\mathrm{U}_{\text {res }} / \mathrm{W}_{\text {res. }}$. Both reflect the proportion of $\mathrm{W}$ either transformed into a stored energy $U\left(U_{\text {res }}\right)$ or into a deformation heat $Q\left(Q_{\text {res }}\right)$ at given $\varepsilon_{\text {def. }}$. The ratios depend on $\varepsilon_{\text {def }}$ and characterize some features of deformation mechanism operating at different stages of a plasticity process. Fig.4 gives the ratio values for several glassy polymers $[4,26]$. The data on $U_{\text {res }}$ storage at low strains, $\varepsilon_{\text {def }}<\varepsilon_{y}$, are presented in Fig. 5 [17, 18].

The mostly striking feature of the results is extremely high ratio of $\mathrm{U}_{\text {res }} / \mathrm{W}_{\text {res }} \approx 100 \%$ at small strains (Fig.5). It appears that such high fraction of $U\left(U_{\text {res }}\right)$ is typical for all polymer glasses. It was suggested earlier, that high level of energy storage in glassy polymers is due to their disordered structure [4]. Crystalline metals store only small fraction (few \%, [15]) of W. (At impact loading of crystalline metals the ratio may increase up to $20-25 \%$ [20], but it is still considerably lower than for polymeric glasses). The expended deformation work is transformed mainly into heat in metals. High U/W ( $\left.\mathrm{U}_{\text {res }} / \mathrm{W}_{\text {res }}\right)$ ratio in glassy polymers manifests that all expended $\mathrm{W}$ is converted into deformation defect production process. Nothing is going into $Q$. This feature of plastic deformation of glassy polymers reflects the specific character of their plastic behavior. We do not know now any other materials with such high ratio of $\mathrm{U} / \mathrm{W}$.

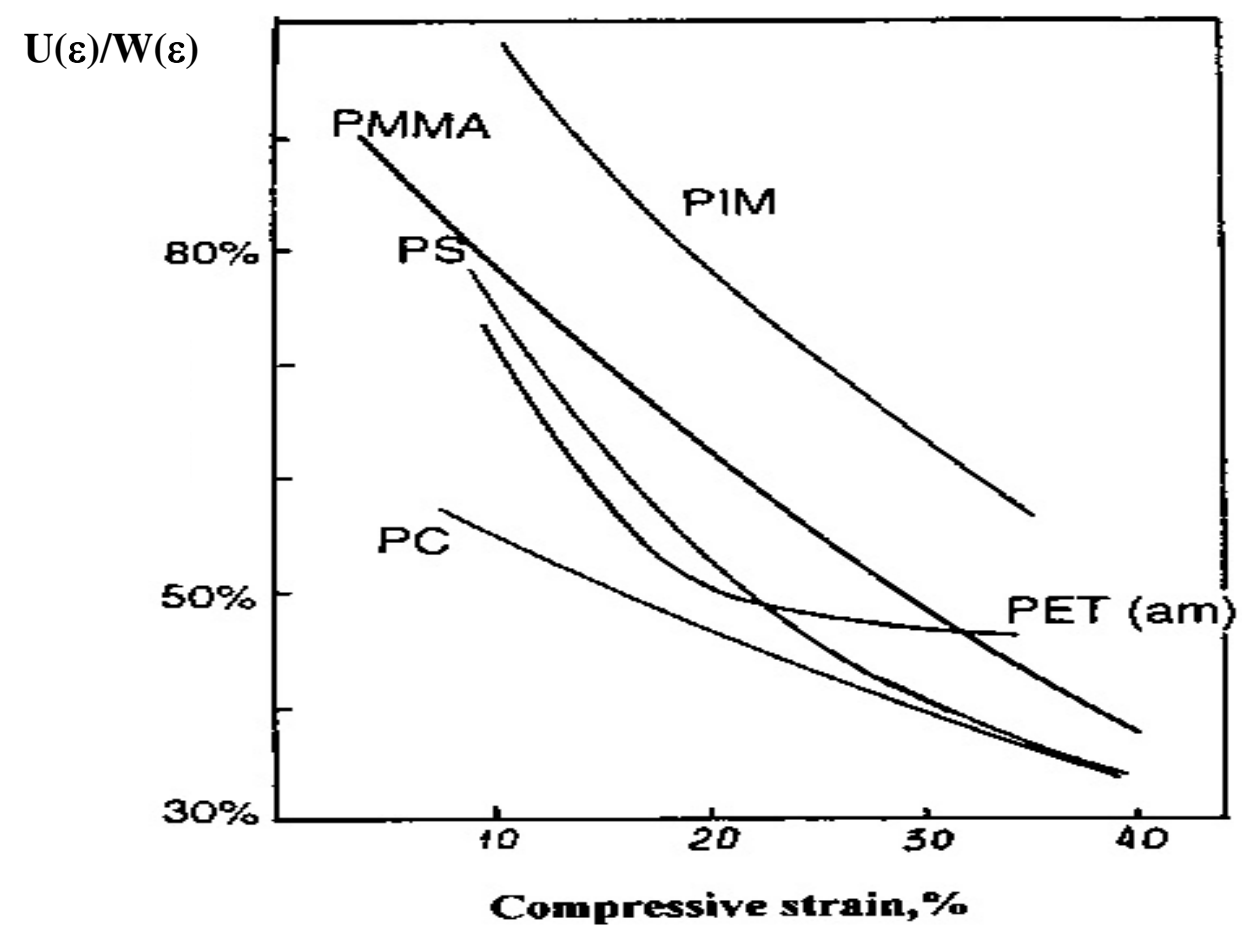

Fig. 4. Ratio $U(\varepsilon) / W(\varepsilon)$ for different glassy polymers for strains $\varepsilon_{\text {def }} \geq \varepsilon_{y}[4,26]$. $T_{\text {def }}=$ room temperature.

To check the correctness of our measurements and compare the behavior of crystalline metal and glassy polymer we had performed deformation of $\mathrm{Cu}$ single crystal in the same DC instrument and the same straining conditions [17]. For $\mathrm{Cu}$ it was found that practically all expended W was transformed into deformation heat $Q$. 
It appears from the earliest stages of the non-elastic processes. Such behavior of $\mathrm{Cu}$ was expected from published data [15] and shows that results of our measurements with polymers are correct. Results shown in Fig. 4 and 5 clearly demonstrate serious differences existing in non-elastic deformation mechanisms operating in crystalline metals and glassy polymers.
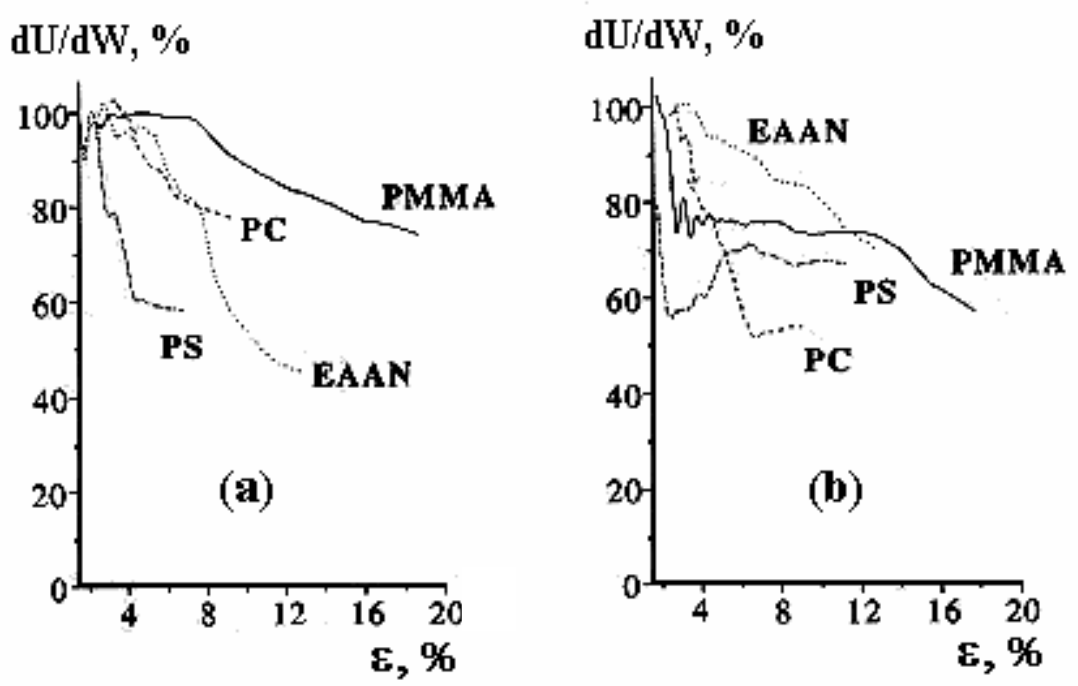

Fig. 5. The ratio dU/dW in the deformation of glassy PC, PMMA, EAAN and PS for $\varepsilon_{\text {def }} \leq \varepsilon_{y}[17,18]$. (a)-annealed, (b)-quenched samples. $T_{\text {def }}=$ room temperature.

A high value of $\mathrm{U} / \mathrm{W}$ for glassy polymers, especially at the beginning of non-elastic processes, gives the possibility to create several conclusions. (1) Chain uncoiling can't be the rate-controlling process in non-elastic deformation of glassy polymers at early deformation stages. Whole W $(100 \%)$ is accumulated in a sample when chain uncoiling (change of the conformational set of chains) had not appeared yet. The phenomenon of the energy of cold work accumulation should relate to a process of some other than chain uncoiling nature. Our suggestion is: whole mechanical work in the beginning of an inelastic deformation process of organic polymer glasses is expended for nucleation of strain carriers. These plasticity carriers are responsible for further development of the deformation process in glass, for steady-state flow, for example. It means that nucleation of plasticity carriers is the rate-controlling process of glassy polymers plasticity. The situation resembles the plasticity of crystals [36, 37], where initial stage of loading is related to nucleation of dislocations and the dislocations glide over easy glide planes then $[8,9,36]$. It appears that similar nucleation of plastic carriers happens in polymer glasses also. However, the carriers in glasses can't be the same nature as in crystals [37]. Possible nature of such carriers was discussed in [1, 4]. (2) High values of the ratio $\mathrm{U}(\varepsilon) / \mathrm{W}(\varepsilon)=80-30 \%$ still are conserved in polymer glasses at higher than $\varepsilon_{y}$ strains (Fig.4), up to $\varepsilon_{\text {def }}=40-50 \%$ at least. We believe the situation manifests a high importance of nucleation events after yield point deformation also.

It is interesting to note that comparatively rigid-chain polymers such as PC and aromatic polyesters ("Vectra ${ }^{\mathrm{TM}}$ " for example) needs less mechanical work than many flexible chain polymers for development of plastic flow [26]. It shows that the plastic resistance of rigid-chain glassy polymers is lower than many flexible chain polymer glasses. If rate-controlling step of the deformation process would be chain uncoiling 
one should expect the opposite behavior for the flexible and rigid-chain glassy polymers.

Recovery of $u_{\text {res }}$ and $\varepsilon_{\text {res }}$ in the deformed polymer glasses

To understand deformation mechanisms operating in glassy polymers one should relate the stored energy $U(\varepsilon)$ and $U_{\text {res }}\left(\varepsilon_{\text {res }}\right)$ to the particular strain components coexisting in a deformed sample. It is commonly accepted [10-14] that moderate and large strains in macromolecular compounds appear from chain uncoiling which is going trough conformational transformations in coils during deformation. Such point of view presumes that the energy storage in a cold work of polymer glasses should be related to the conformational component of $\varepsilon_{\text {def. }}$ It is also suggested that the conformational uncoiling of chains is the rate-controlling step in plastic deformation of polymer glasses. Nearly all models of plasticity of glassy polymers also are putting chain uncoiling as the rate controlling process [10-14]. Such deformation behavior of glassy polymers is called the "forced high elasticity" [10].

However, experimental results on the $U_{\text {res }}$ storage bring some doubts for validity of the picture. Conformational rearrangements undoubtedly control chain uncoiling processes in rubbers. However, rubbers do not store the deformation energy $U_{\text {res. }}$.

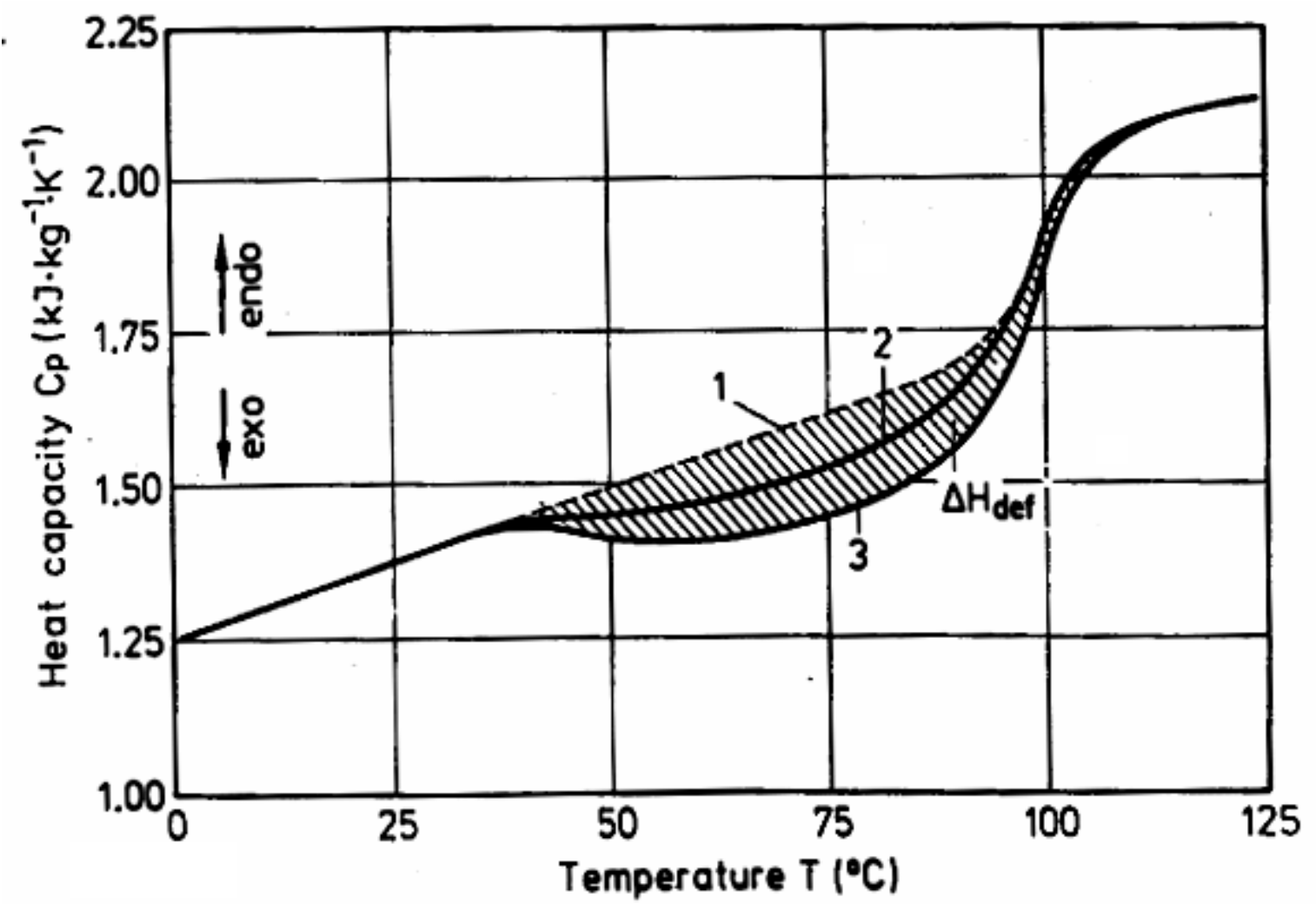

Fig. 6. DSC traces for glassy PS deformed in different ways [38]. Heating rate - 20 $\mathrm{deg} / \mathrm{min}$. 1- Non-deformed sample. 2- Uniaxial tension, $\varepsilon_{\text {res }}=3 \%$. 3-Uniaxial compression, $\varepsilon_{\text {res }}=30 \%$. $\mathrm{T}_{\text {def }}=$ room temperature .

In glassy polymers the stored $U_{\text {res }}$ may be erased from a sample by heating it from $\mathrm{T}_{\text {def }}$ through $\mathrm{T}_{\mathrm{g}}$ interval, as it was described in the previous section. Complete recovery of initial sample shape, size and $U_{\text {res }}$ happens for nearly all glassy polymers at heating. Such recovery happens for crystals also, polymeric and non-polymeric. Only high temperature border of the crystal's heating is a little higher than melting 
temperature $T_{m}[21,22]$. The thermally stimulated recovery (TSR) technique gives very important information about the nature and features of $U_{\text {res }}$ and $\varepsilon_{\text {res }}$ accumulated in the solids $[4,39]$.

Fig. 6 demonstrates behavior of PS samples deformed in different ways [38]. Curve 1 is a standard DSC trace for the non-deformed sample. Softening manifests itself through the endothermic jump of $C_{p}$ in $T_{g}$ region (about $100^{\circ} \mathrm{C}$ ). Very different situation appears after room temperature deformation of the glassy sample. Material deformed by uniaxial tension (curve 2) and compression (curve 3 ) demonstrate at heating the "exothermic DSC anomaly" which cover in this case the temperature range from $\approx 35^{\circ} \mathrm{C}$ up to the upper boundary of the softening interval. It was suggested earlier that the "exothermal anomalies" are related to the release of the stored deformed sample energy $U_{\text {res }}$ during heating [40]. Later measurements with different methods have supported this point of view [41, 42].

Crucially important features of the deformation and recovery processes manifest themselves in the following measurements. $U_{\text {res }}$ is released from a deformed sample mainly in the glassy state of a material, at temperature interval from $\approx T_{\text {def }}$ up to the beginning of softening interval. The main fraction of $U_{\text {res }}(\approx 80 \%)$ is recovered below $T_{g}$. Only $\approx 20 \%$ of the stored $U_{\text {res }}$ is recovered in $T_{g}$ interval, as it clearly seen for $P S$ (Fig.6, area under the recovery peak at glass transition region in comparison with the exo- anomaly peak area in glassy material below $\mathrm{T}_{\mathrm{g}}$ ). Experiments had shown the lower $T_{\text {def }}$ is, the lager fraction of $U_{\text {res }}$ is recovered below $T_{g}[4,38]$. These results show that it is difficult to refer such $U_{\text {res }}$ release to conformational recovery, especially at considerably low recovery temperatures, $100-300^{\circ}$ below $\mathrm{T}_{\mathrm{g}}$. The situation indicates the process in which carriers of a non-elastic strain are nucleated mainly in the glass and may exist only in this state of matter.

The next thermally stimulated experiments were performed for $\varepsilon_{\text {res }}$ recovery (Fig. 7). Recovery rate $\dot{\varepsilon}_{\text {res }}$ is represented for EAAN sample deformed at two different temperatures [38]. There are two recovery rate curves: the first (curve 1) appears for the sample deformed at $160^{\circ} \mathrm{C}$, above it's $\mathrm{T}_{\mathrm{g}}=145^{\circ} \mathrm{C}$ [32] and cooled then to room temperature at the condition $\varepsilon_{\text {res }}=$ const. and unloaded. In the second case (curve 2) the sample was deformed at room temperature. Both, samples containing $\varepsilon_{\text {res }}$ were heated than (Fig. 7) from $T_{\text {room }}$ through the $T_{g}$ interval.

The shapes of recovery curves are very different. Size recovery of the sample 1 happens only in the $\mathrm{T}_{\mathrm{g}}$ interval, but the sample 2 shows two recovery processes (peaks), one in the $\mathrm{T}_{\mathrm{g}}$ interval and the other broad recovery peak below $\mathrm{T}_{\mathrm{g}}$. Comparison of the Fig. 6 and 7 immediately shows that the low temperature recovery, the LTR peak in the glassy state of the material, carry main fraction of the stored $U_{\text {res. }}$.

What is the strain $\varepsilon_{\text {res }}$ component recoverable below $\mathrm{T}_{\mathrm{g}}$ ? It is definitely anelastic (delayed elastic [1]) strain component. Only this component of $\varepsilon_{\text {res }}$ is able to recover in the solid state of matter, i.e. below $\mathrm{T}_{\mathrm{g}}$ in glasses. All strains existing in glass and able to disappear in the solid state of matter belong to the deformation defects whose recovery barriers are lower than $\mathrm{kT}_{\mathrm{g}}$ ( $\mathrm{k}$ is the Boltzmann constant). The strain recoverable only after material softening during its heating will be called here the plastic $\left(\varepsilon_{\mathrm{pl}}\right)$ component. 


\section{Recovery rate, $\% / \mathrm{min}$}

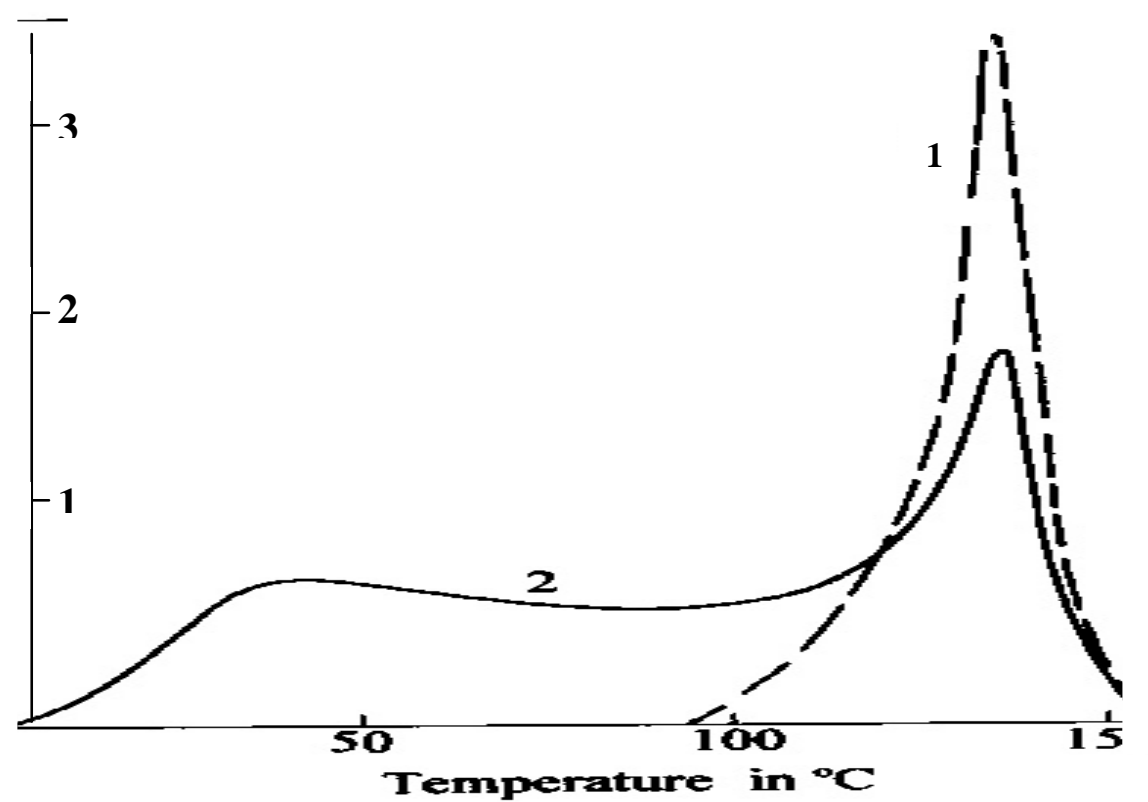

Fig. 7. Thermally stimulated $\varepsilon_{\text {res }}$ recovery curves for EAAN deformed at different temperatures. $1-\mathrm{T}_{\text {def }}=160^{\circ} \mathrm{C}, \varepsilon_{\text {res }}=17 \%$. Deformed sample was cooled then to room temperature with fixed length $\left(\varepsilon_{\text {res }}\right.$ constant). $2-T_{\text {def }}=$ room temperature. $\varepsilon_{\text {res }}=20 \%$. Recovery rate $\dot{\varepsilon}_{\text {res }}-$ in \%/min. Heating rate $-20 \mathrm{deg} / \mathrm{min}[16]$.

In other words, the true plastic component of strain is able to recover only in the liquid (rubbery) state of matter. From the definitions one has to accept that the main strain component in glassy polymers carrying $U_{\text {res }}$ is the anelastic component $\varepsilon_{\text {an }}$. Important features of the component are considered in $[1,43]$. In polymers $\varepsilon_{\mathrm{pl}}$ may contain two parts, $\varepsilon_{\text {conf }}$ and $\varepsilon_{\mathrm{vp}}$ (see the caption to Fig. 1). Only $\varepsilon_{\mathrm{vp}}$ is real permanent strain and for it's recovery a sample should be heated up to $\approx \mathrm{T}_{\text {flow. }}$. The conformational component of the deformed glassy material is recovered in $\mathrm{T}_{\mathrm{g}}$ interval.

From Fig. 7 it clearly seen that the high temperature recovery (HTR) component is physically related to the process of restoration of conformational violations which had appeared in a glassy sample during its loading (curve 1). Chain uncoiling is the dominating deformation micro-process in the rubbery state of polymers. But it is not so in glassy polymers. In the unloaded and glassy material containing $\varepsilon_{\text {conf }}$ this strain component cannot be recovered at temperatures sufficiently below $T_{g}$ because any conformational rearrangements are frozen in a glass at low temperatures.

Fig. 8 demonstrates recovery curves for PC deformed at $20^{\circ} \mathrm{C}$ up to different strain $\varepsilon_{\text {def }}$ and $\varepsilon_{\text {res }}$ levels. One may see that at small $\varepsilon_{\text {res }}$ only the LTR peak appears at heating. No strain is recovered at $T_{g}$. It means that there is no conformational strain component in the glassy PC deformed up to small strains. The same is true for all studied glassy polymers. Recovery peak at $T_{g}$, (HTR peak) appears only in samples deformed at room temperature in the glassy state up to $\varepsilon_{\text {def }}>6-7 \%$.

Existence of $U_{\text {res }}$ (Fig. 6) and $\varepsilon_{\text {res }}$ in the deformed and unloaded glassy polymers much below $T_{g}$ manifests that main part of the energy storage in glassy polymers is not related to conformational uncoiling of deformed chains. The stored energy $U_{\text {res }}$ in 
glassy polymers below $\mathrm{T}_{\mathrm{g}}$ is carried by anelastic strain component, $\varepsilon_{\text {an }}$. The mechanical production of the carrying energy deformation defects in a glass should be related not to specific polymeric process (chain uncoiling), but to the process of nucleation of local shear transformations. It seems that such nucleation of local shear transformations is the process characteristic for any disordered solid. The computer modeling given below supports such suggestion.

By erasing the LTR component from a deformed glassy material one may prepare a deformed sample containing only the conformational component of $\varepsilon_{\text {res }}$ [4], but not $\varepsilon_{\text {an }}$ (only HTR peak appears at heating, Fig.9, curve 4). Let us now deform again this sample at room temperature. New deformation gives the following result: the LTR strain component $\left(\varepsilon_{\text {an }}\right)$ appears first and grows up. There is no increase in the intensity of the existing HTR peak yet. The behavior of HTR peak shows that the number of excited conformers in a sample is not growing at the beginning of the second deformation. The HTR peak intensity starts to increase at the moment when the $\varepsilon_{\text {an }}$ (area under the LTR peak) reaches saturation. Only further loading increases intensity of the HTR peak, and the intensity of the LTR peak stay constant during such grows of the HTR peak [4].

\section{Recovery rate,\%/min}

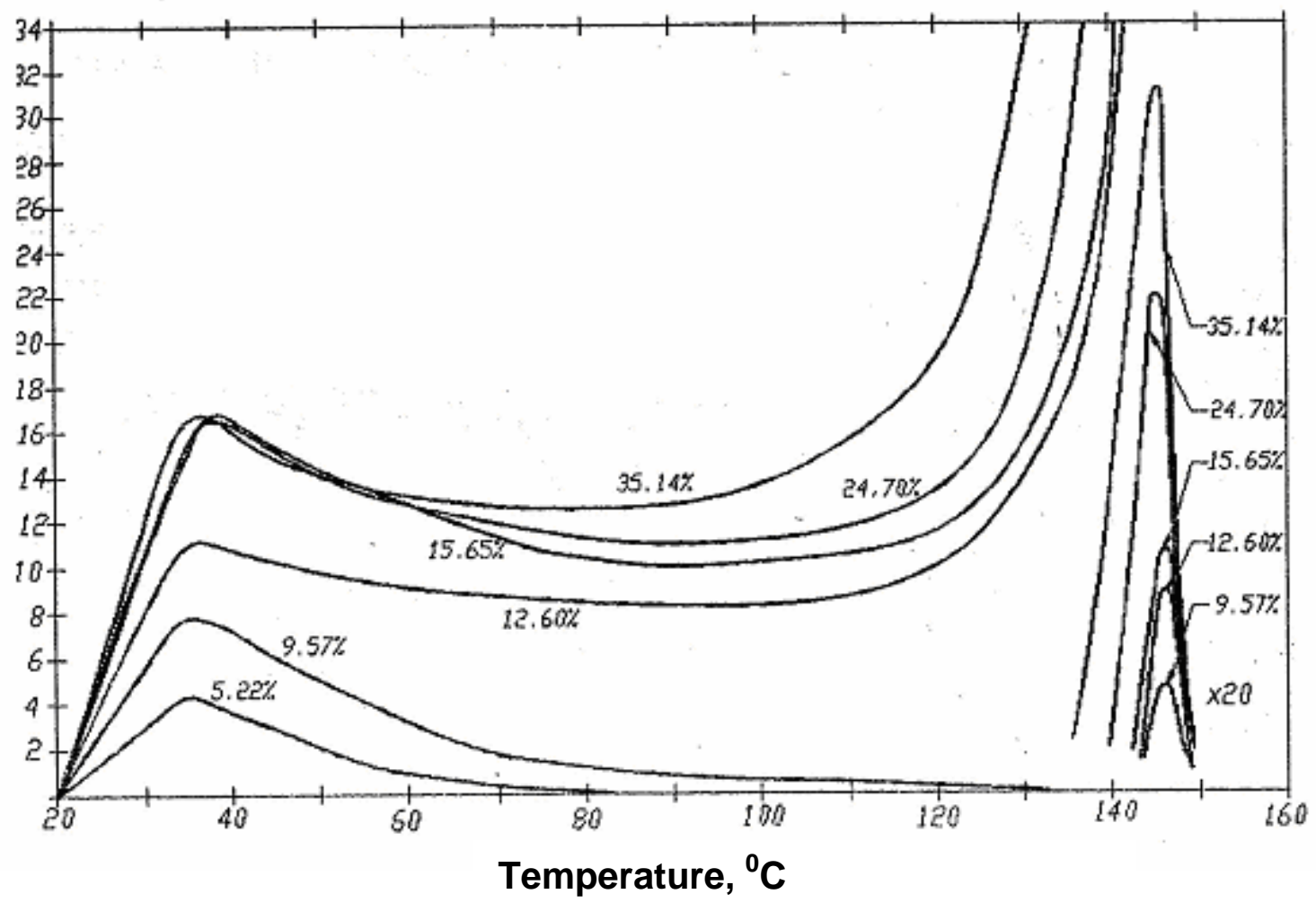

Fig. 8. Thermally stimulated rate recovery curves for $\mathrm{PC}$ deformed at $20^{\circ} \mathrm{C}$ up to different strain levels. Numbers on curves show amount of strain $\varepsilon_{\text {res }}$ for each sample $\left(\mathrm{T}_{\text {def }}=\mathrm{RT}\right)$. Amount of $\varepsilon_{\text {def }} \approx\left(\varepsilon_{\text {res }}+5-7 \%\right)$. Heating rate $-20 \mathrm{deg} / \mathrm{min}$. Intensity of recovery peaks at $T_{g}$ multiplied $x 20$.

The situation is typical for all studied glassy polymers. The results clearly show that $\varepsilon_{\text {an }}$ is the necessary pre-cursor to any conformational rearrangements appearing in deformation of polymer glass. The $\varepsilon_{\text {an }}$ component appears in polymer first, and only 
later chain uncoiling happens and $\varepsilon_{\text {conf }}$ appears. New chain conformers can't appear in glass without nucleation of $\varepsilon_{\text {an }}$ carriers. It means that the mechanisms of nonelastic deformation operating in glassy polymers are very different in comparison with rubbers. In rubbery polymers chain uncoiling happens directly under acting of an external force. Such process is not active in the glassy material. The deformation in glasses proceeds in two stages: the first one is the nucleation of anelastic strain transformations (AST) of non-conformational origin. The second process is the spontaneous transformation of the ASTs into energetically less excited local structures. Such transformation always goes through local conformational rearrangements producing chain uncoiling. And deformation heat $Q$ appears due to such spontaneous transformations of ASTs. The way of the non-elastic deformation heat production in glassy polymers is different in comparison with crystals. This is the general physical picture of non-elastic deformation of polymer glasses. The picture embraces all experimental facts shown above and existing in literature.

\section{Recovery rate, \%/min}

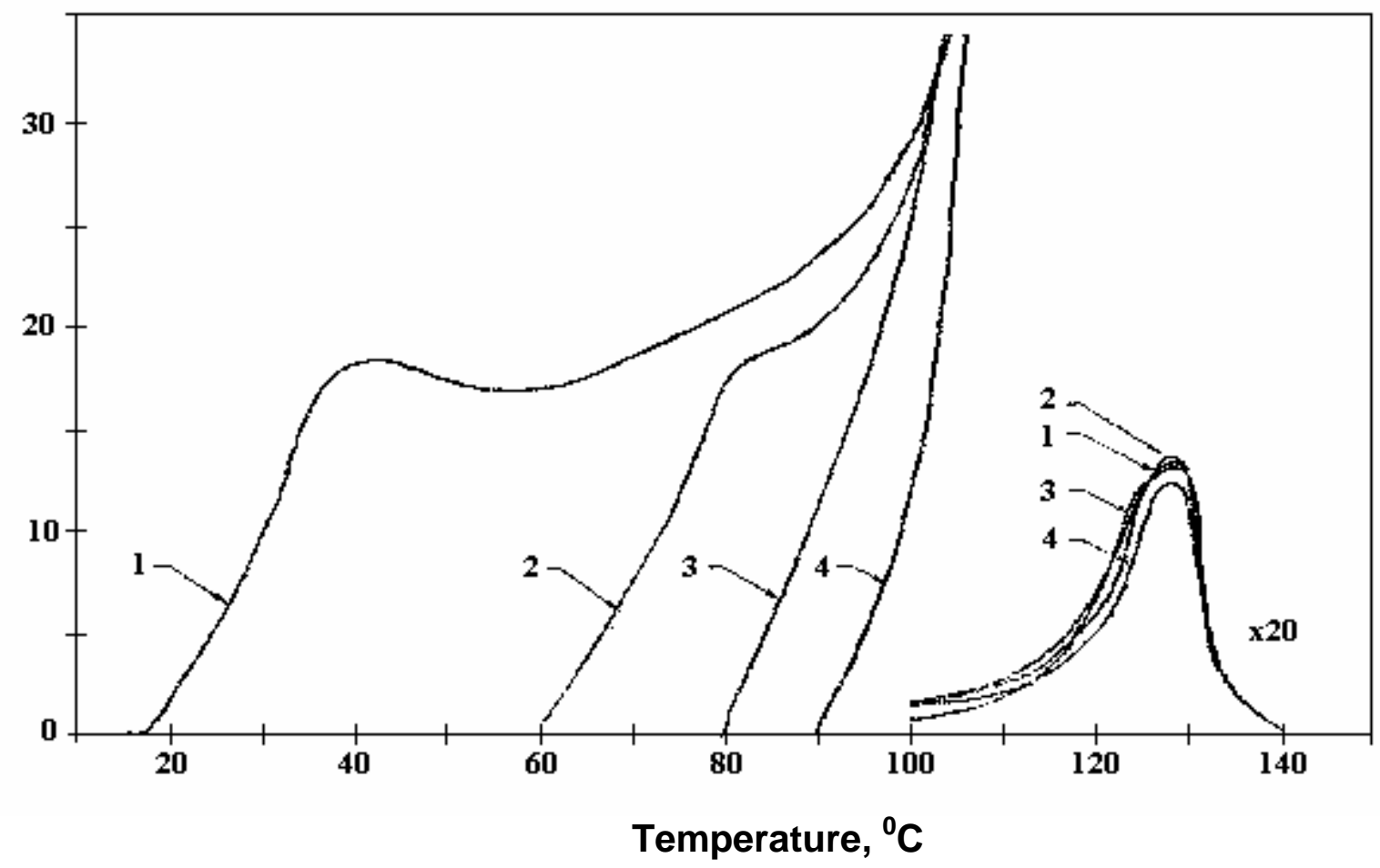

Fig. 9. 1.Thermally stimulated $\varepsilon_{\text {res }}$ recovery rate curves for PMMA, annealed and deformed at $\mathrm{T}_{\text {room }}$ by compression to $\varepsilon_{\mathrm{res}}=27.8 \%$. 2 - 4. Washing out of the LTR peak by heating of sample 1 up to different temperatures. 2 - PMMA deformed at $\mathrm{T}_{\text {room }}$ up to $\varepsilon_{\text {res }}=27.8 \%$ and kept at $60^{\circ} \mathrm{C}$ for $5 \mathrm{~min}$. Final $\varepsilon_{\text {res }}=26.1 \% .3-\mathrm{PMMA}$ deformed at $T_{\text {room }}$ up to $\varepsilon_{\text {res }}=27.8 \%$ and kept at $80^{\circ} \mathrm{C}$ for 5 min. Final $\varepsilon_{\text {res }}=24.3 \%$. 4- PMMA deformed at $\mathrm{T}_{\text {room }}$ up to $\varepsilon_{\text {res }}=27.8 \%$ and kept at $90^{\circ} \mathrm{C}$ for 5 min. Final $\varepsilon_{\text {res }}$ $=22.1 \%$. Intensities of the HTR peaks are multiplied x20. Recovery rate $\dot{\varepsilon}_{\text {res }}-$ in $\% / \mathrm{min}$. Heating rate $-20 \mathrm{deg} / \mathrm{min}$.

\section{Computer modelling of the deformation of atomic glass [44, 45]}

For structurally complex systems, such as glasses, computer modeling often helps to clarify details of local rearrangements occurring in them under acting of external stimuli. A good example of such study is structural transformations occurring during 
deformation. To visualize the process of nucleation and development of the anelastic strain carriers we have investigated a simple atomic glassy system subjected to an external shear force. We tend to simplify the chosen system, and therefore we did not consider polymer chains in the calculations. We wanted to introduce to the system only two important factors: disorder of atomic positions and the solidity. Twodimensional (2D) system was chosen for the sake of simplicity. To create disorder two types of "atoms" with different diameters and masses were chosen for construction of the working system. Fig. 10 gives the image of the initial model system [44, 45]. Diameters of atoms were 1 and 1.5 and masses 15 and 30 a.m.u.

It is known [46] that systems with such ratio of atomic diameters can't crystallize. The system contained 239 large and 269 small particles. We have performed molecular dynamic calculations with the Lennard-Jones inter-particle interactions.

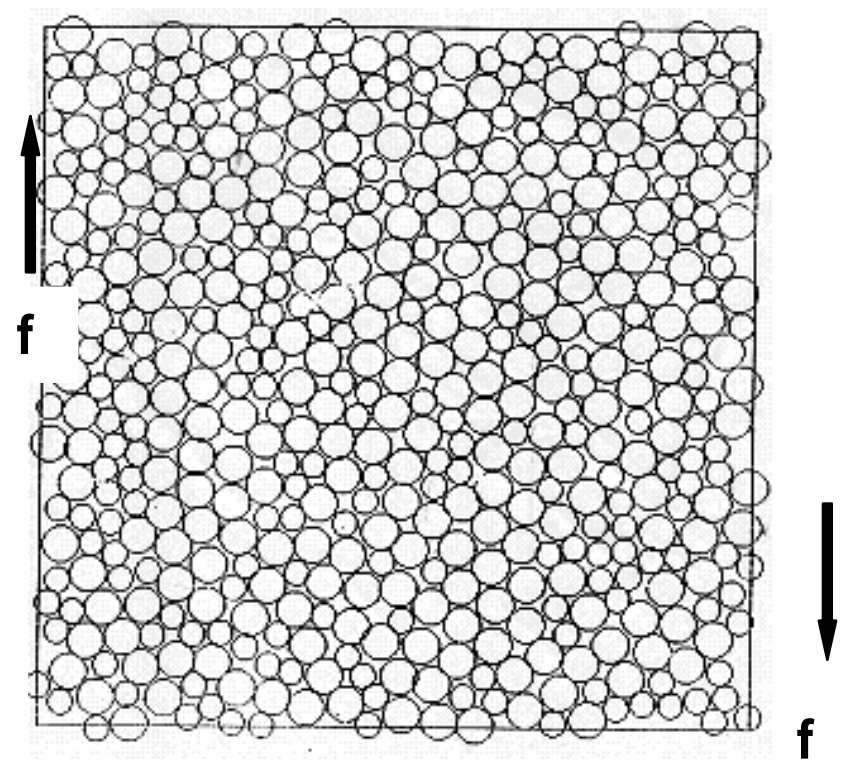

Fig. 10. The system for computer modeling of deformation. Glassy state. $T_{\text {def }}=0.2 T_{g}$. Packing density 0.73. f- applied shear forces [44, 45].

Time step in the calculations was about 0.1 picosecond. We simulated broad temperature range covering the system behavior from the liquid (high temperature) to the solid glassy state (low temperatures) through the glass transition interval [44, 45].

Straining of the system was performed by shear forces $f$ applied in a way shown in Fig. 10. "Rigid wall" boundary conditions were used in this case [44, 45]. Change of the macroscopic shear strains $\gamma$ for different level of forces are demonstrated in Fig. 11. One can see that studied computer glass shows macroscopic stress-strain behavior typical for solids. At small forces the system response is linear-elastic and reversible at unloading (up to $\mathrm{f}=10-15 \mathrm{~N}$ ). Than plastic flow occurs and macroscopic residual strains $\gamma_{\text {res }}$ stay in the sample after unloading.

Analysis of accumulation of $\gamma_{\text {res }}$ with time (number of computing steps) shows $[45,47]$ that strain is growing systematically with time. However, at high forces $(f=24-32 \mathrm{~N}$ ) some local minima appear at the curves. Sample unloading upon such minima shows that the macroscopic strain is increased there by jumps. Such behavior of sample demonstrates that some pronounced deformation events happen in the vicinity of $\gamma_{\text {res }}$ minima. 


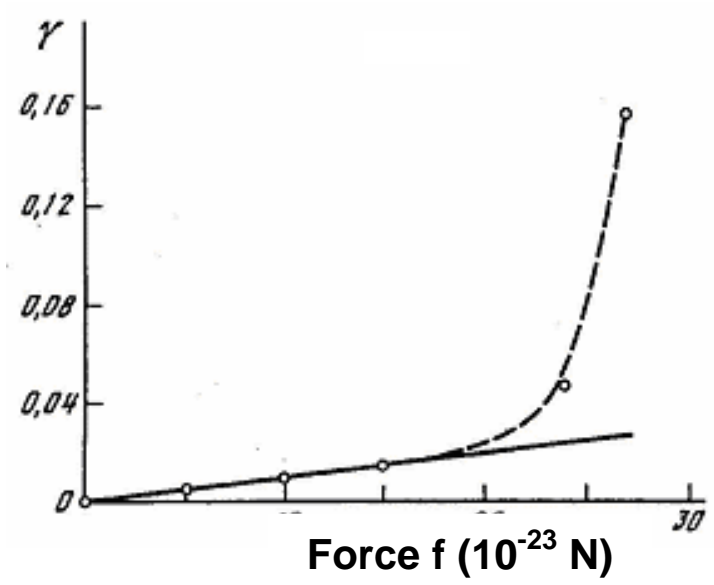

Fig. 11. Macroscopic shear strain $(\gamma)$ - force (f) curve for computer glass shown in Fig. 10.

We analyzed the deformation events, the local atomic rearrangements at these $\gamma_{\text {res. }}$. Fig. 12 represents the results. Initial system was loaded as it shown in Fig. 10 and the pattern 12a represents the first inelastic deformation step in the system. All area of the sample was subjected to Delaney tessellation. Shown triangles give local space belonging to each atom. Changes of shape and size of the triangles under load produce amount of strain accumulated around each atom.

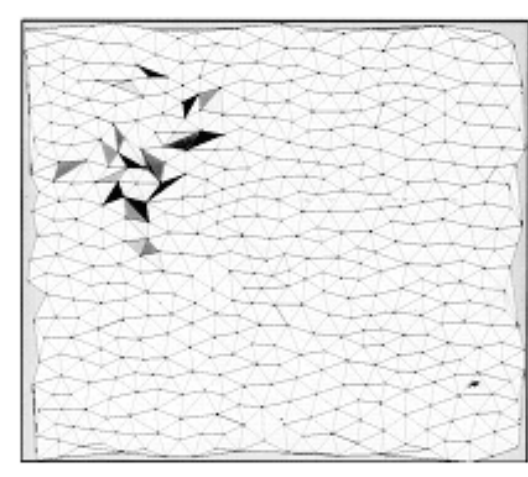

A

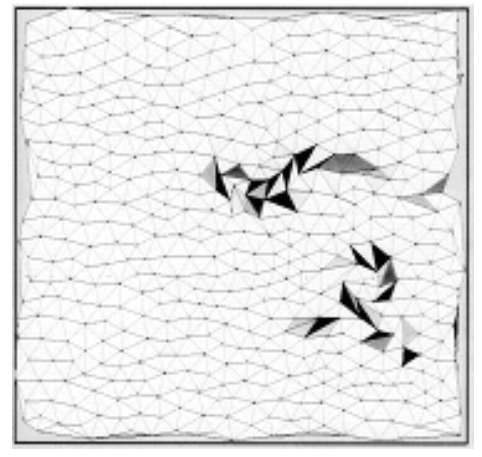

B

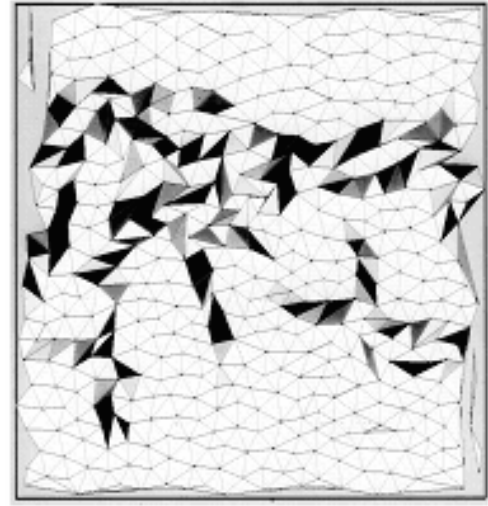

C

Fig. 12. Stages of the inelastic deformation in the computer glass. Points show centers of atom's location. A-the first localized shear event appears. B-the second localized shear event appears at new location. $C$-the third and may be the forth and fifth events appear. All the events unify and form the shear band crossing entire sample [45]. Colors in the triangles give amount of local shear strains: black- $\gamma_{\text {loc }} \approx$ 1.2 , gray $-\gamma_{\text {loc }} \approx 0.12$, light grey- $\gamma_{\text {loc }} \approx 0.05$, white $\gamma_{\text {loc }} \leq 0.03$.

The first event corresponds to: $f=24 \mathrm{~N}$, macroscopic $\gamma_{\mathrm{res}} \approx 0.03$. Shear strains in the event are much higher than the macroscopic one. The number of computing steps is $\approx 1200$. Fig. $12 \mathrm{~B}$ and $\mathrm{C}$ represent the second and third events $(\mathrm{f}=24 \mathrm{~N}$, macroscopic $\gamma_{\text {loc }} \approx 0.05$, number of computing steps $\approx 3000$ and $f=32 \mathrm{~N}$, macroscopic $\gamma_{\text {loc }} \approx 0.12$, and the number of computing steps $\approx 3200$ correspondingly). Local strains in the second and the further events again are much higher than macroscopic strains. 
Analysis of energy distribution had shown that energy excess is also localized in the vicinity of shown ASTs. Dilatational component of strain is close to zero. It shows that the energy excess around ASTs has the elastic, not voluminal origin.

Several important features of glassy material plasticity one may find from the modeling. The first elementary deformation events appear locally. Only about 20-25 atoms are involved in the individual shear transformation. The rest of a sample stays deformed a little, only linear-elastically. The second deformation event appears at new, separate location of the sample. It changes a little the distribution of strains around the first event. However, both events look like independent deformation steps. Namely, such kind of shear transformations we consider as the anelastic shear transformations (AST) in glassy material.

The picture demonstrates that there is no expansion (growth) of the ASTs during loading. Such behavior for glasses was suggested in [37]. Local strains $\gamma_{\text {loc }}$ inside of the events are much higher than the macroscopic $\gamma_{\text {res. }}$. The picture shows that the non-elastic deformation behavior of the disordered system is very different in comparison with crystals where the nucleated dislocation are expanding under action of external load by glide trough easy glide planes $[36,37]$. It shows also that a nucleation of locally strained sites in disordered structures is the main deformation process at early loading stages in disordered solids. The same conclusion had appeared from the experiments with glassy polymers shown above.

Analysis of other parameters of computed deformation for both deformation events had shown:

1. The highest stresses are concentrated locally around the localized strain transformations (events 1 and 2),

2. $U_{\text {res }}$ is also concentrated locally around the events; it decay to zero at distances about $20 \AA$ from the center of the first and the second events,

3. Any specific non-crystallographic atomic configurations (such as 5- or 7-numbered polygons were not found in sites where the first and the second ASTs had appeared.

The 3-rd deformation event (Fig. 12C) looks quite different in comparison with the previous. Kind of macroscopic deformation band appears in the sample. Because we did not see any expansion of the first and the second events we suggested that the third and may be forth and fifth events were nucleated in the sample. Following merging all the previous and new ASTs united all local strains into one continuous macroscopic structure. It looks like some co-operative deformation rearrangement.

The computer modeling gave many interesting details of the development of plastic deformation in a disordered solid. The deformation process starts from nucleation of local shear events. Such events store the energy of cold work, which well coincide with the picture withdrawn from the experimental data. Clearly, the nucleation of local ASTs is the main step in plastic deformation of disordered solids at early loading stages. Such local transformations are united then.

The presented oversimplified model can't reflect deformation details of macromolecular glasses. However, it gives many important deformation features, possibly common for any non-covalent glasses, polymeric, metallic and low molecular mass organic.

\section{Physical picture of non-elastic deformation for glassy polymers}

Experiments and computer modeling results combined together permits to formulate an appropriate general picture of plastic response for glassy polymers. Such picture is shown in Fig. 13. 
Loading of glassy sample raise macroscopic $U_{\text {res }}$ of an entire sample and creates the anelastic shear transformations with the excess of local energy $U_{\text {loc. }}$. Growth of the concentration of such ASTs provides an increase of the stored energy of cold work in a sample. Accumulation of shear transformations together with accumulation of $U_{\text {res }}$ is represented by the Path 2 in the scheme (Fig. 13).

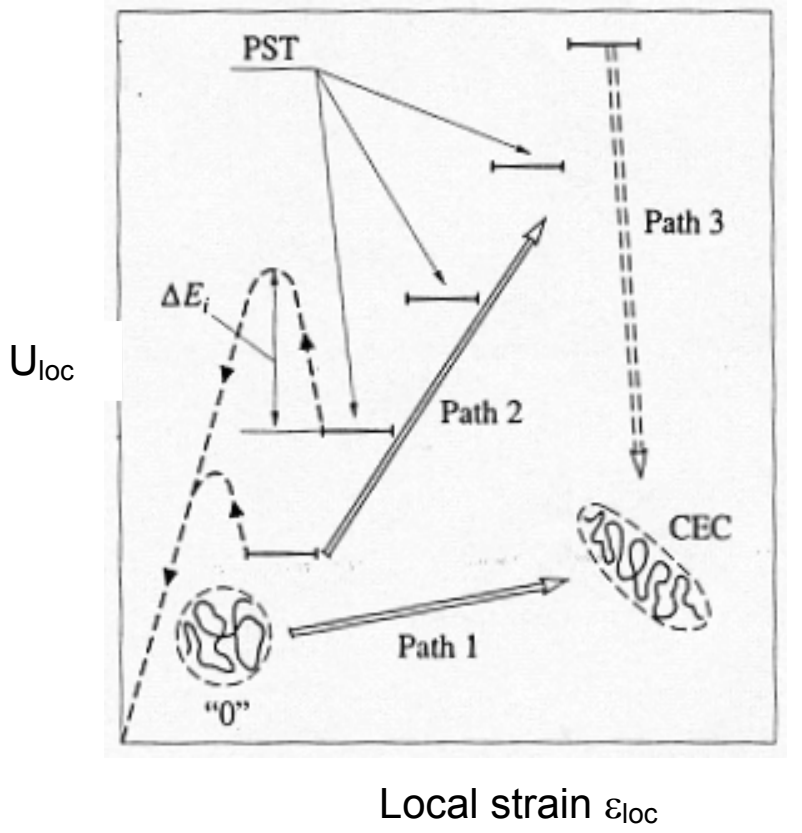

Fig. 13. Scheme of non-elastic deformation of the rubbery (Path 1) and glassy (Path 2) polymers [4, 28 32, 38]. "0"- initial macromolecular coil. CEC-the conformationally excited coil, enriched by the extended conformers appearing upon straining. PSTthe localized plastic shear transformations carrying energy excess $U_{\text {loc }}$. PSTs accumulated by a glassy sample carry the total $U_{\text {def. }} E_{i-}$ activation barrier of recovery of $\mathrm{PST}_{i}$. Path 3 - the spontaneous relaxation of the highly excited PSTs. $\mathrm{U}_{\mathrm{loc}}$ is decreased during the relaxation.

The Path 1 represents deformation of rubbery polymer through chain uncoiling. There is some increase of $U$ for the deformed and loaded sample (but $U_{\text {res }}$ does not increase, because the deformation energy excess disappears completely in unloaded rubbers). The $U$ increase in rubbers is related to changes of coil sizes going through appearance of new, extended conformers in macromolecules. Such growth of $U$ is much smaller than in non-elastic deformation of solid polymers $[4,28,32,38]$.

Experiments show the saturation of $U_{\text {res }}$ growth in polymer glasses. It suggests an appearance some relaxation processes decreasing $U_{\text {loc }}$ and total $U_{\text {res. }}$. Such relaxation should go microscopically through the energy decrease of some ASTs. We suggested that such $U_{\text {loc }}$ relaxation is going in the stage of the AST's unification. Clearly, the larger the shear, the lower is its $U_{\text {loc. }}$. However, such unification of ASTs should violate local conformational structure of macromolecules. Otherwise the decrease of local energy cannot fit the growth of local and total strains. This stage of the deformation process is represented by the Path 3 in the scheme (Fig. 13). A local strain $\varepsilon_{\text {loc }}$ does not relax together with $U_{\text {loc. }}$ It is only transformed from the anelastic (plastic) into conformational strain component. This is the way of chain uncoiling. Important point to note is that such uncoiling is not the main rate controlling process in plastic deformation of polymer glasses. This uncoiling is just the fitting of a macromolecular compound to the process of AST's nucleation in glassy material. Local anelastic strain in a relaxing AST becomes smaller, but local conformational 
strain is growing. It happens during relaxation of each AST. Such picture reflects the fact that any event of chain uncoiling can't happen without the relaxation of an existing AST. Therefore, macroscopic deformation of polymer glasses always starts from the nucleation of ASTs. Further transformations of the ASTs define the following deformation process, the steady flow and steady energy storage. Presented picture is differed seriously from current models of plastic deformation of glassy polymers.

Local conformational changes are important in the total process of glassy polymer's deformation. In our view they are responsible for the deformation heat $Q$ appearance in polymer glasses. The deformation heat arises from the energy decrease in each relaxation of $\varepsilon_{\text {loc }}$ and amount of the released heat $Q_{\text {loc }}$ depends on concentration of relaxing ASTs per time unit. Such picture of $Q_{\text {def }}$ appearance is completely different in comparison with crystals.

Other significance of conformational changes in polymer glasses is the following. They produce local orientation and final texture in deformed sample. Orientation and texturing of glassy sample is going through small shear steps, transformations and relaxations happening in each shear event. Such picture also manifests that chain uncoiling cannot be the rate controlling process in plastic deformation of polymer glasses. Conformational rearrangements are happening easy and fast. Rate controlling step in non-elastic deformation of glassy polymers, and probably in all non-covalent glasses is the nucleation of new shear events without which any large deformation in glassy material is impossible.

Presented picture fits well for all experimental facts existing in a field. It contains only several suggestions (like deformation heat production and the way of new conformations appearance during an AST energy relaxation). We can't provide now a reasonable mechanism for such events, but the proposed suggestions do not violate whole deformation picture.

Summarizing the paper we would like to emphasize that there are several new features of the proposed model, which break up some customary picture of plastic deformation in polymer glasses. First of all, experiments and computer simulations have shown that the main, rate-controlling step in deformation of disordered solids is the nucleation of anelastic shear transformations. Chain uncoiling exists in deformation process but is not controlling it.

The second point is the serious difference in deformation mechanisms operating in rubbers and glasses. One is not coinciding with the other. And the third point exists. Often the mechanical behavior of polymer materials is related to the initial structure of the polymer (so called correlations between structure and properties). However, in our view such a way is incorrect for mechanical response of polymer solids. Straining brings polymer sample to the new thermodynamic state, an excited one. The energy storage manifests it. And following behavior of sample defines not by its initial structure but the new excited structure. Such process as formation of crazes and cracks, relaxations of stress and strain (creep) and many others depends on the concentration and properties of local deformation events existing in a sample. Such approach is not developed yet in polymer physics. However, such developments look important for the field.

\section{Acknowledgements}

This work is financially supported by Russian Foundation for Basic Research (RFBR), Grant \# 05-03-32481 and by the Program \#3 (2006) of the Chemical Division of Russian Academy of Sciences. 


\section{References}

[1] Perez, Jo.; Physics and Mechanics of amorphous polymers. 1998, A.A. Balkema, Rotterdam.

[2] Argon, A.S.; Inelastic Deformation and Fracture of Glassy Solids. Published in "Material Science and Technology", Cahn,; R.W., Hasan,; P., Kramer, E.J.; Eds. 1993, Vol.6. Mughrabi Ed. VCH Publishers. Weinheim, Germany. pp.461-508.

[3] Boyce, M.C.; Haward, R.N.; Deformation of Glassy Polymers. 1997, Ch.5 in the book: The Physics of Glassy Polymers, Haward R.N., Young R.J. Eds. Chapman\&Hall, London.

[4] Oleinik, E.F.; Salamatina, O.B.; Rudnev, S.N.; Shenogin, S.V.; Polymer Science 1993, 34,1532.

[5] Crist, B.; in: Material Science and Technology. 1993, N.Y. VCH, Vol.12, 427.

[6] Argon, A.S.; Inelastic Deformation and Fracture in Oxide, Metallic, and Polymeric Glasses. In: Glass: Science and Technology. 1980, V. 5. Ch.3. 79.

[7] Lin, .L.; Argon, A.S.; J.Mat.Sci. 1994, 29, 2, 294.

[8] Galeski, A.; Progress in Polymer Sci. 2003, 28, 1643-1699.

[9] Oleinik, E.F.; Polymer Sci. C.2003, 45, 7.

[10] Kargin, V.A.; Slonimsky, G.L.; Encyclopedia of Polymer Science and Technology, 1968, N.Y., Willey, 8, 445.

[11] Krausz, A.S.; Eyring, H.; Deformation Kinetics, 1987, J.Willey, N.Y.

[12] Yannas, I.V.; Luise, R.R.; in: The Strength and Stiffness of Polymers, 1983, Zachariades, A.E.; Porter, R.S.; Eds. N.Y, Marcel Dekker, P.255.

[13] Robertson, R.E.; J.Chem.Phys. 1966, 44, 3950.

[14] Brown, N.; Yield Behavior of Polymers. 1983, In: Failure in Plastics. Brostow, W.; Corneliusen, R.D.; Eds. Ch.6. N.Y. Hansen, P.98.

[15] Bever, M.B.; Holt, D.L.; Titchener, A.L.; In: Material Science, 1973, Chalmers, B.; Christian, J.W.; Massalski, T.B.; Eds. 17, 1.

[16] Salamatina, O.B.; Hőhne, G.W.H.; Rudnev, S.N.; Oleinik, E.F.; Thermochimica Acta, 1994, 247,1.

[17] Shenogin, S.V.; Höhne, G.W.H.; Oleinik, E.F.; Thermochimica Acta, 2002, 391,13 .

[18] Shenogin, S.V.; Hőhne, G.W.H.; Salamatina, O.B.; Rudnev, S.N.; Oleinik, E.F.; Polym.Sci. A, 2004, 46, 21.

[19] Eskaig, B.; Helvetica Physica Acta, 1983, 56, 293.

[20] Pavlov,V.A.; Physical Bases of Plastic Deformation of Metals,1962, USSR Academy of Science Publ., Moscow. Ch.5 p.85.

[21] Beck, P.A.; Advances in Physics (A Quarterly suppl. to Phyl. Mag.), $1954,3,11, \mathrm{~s} 2$.

[22] Clarebrough, L.M.; Hargreaves, M.E.; Loretto, M.H.; In: Recovery and Recrystallisation of Metals, 1963, Hummel, L.; Ed. Interscience, 63.

[23] Taylor, G.I.; Quinney, H.; Proc.Roy.Soc. A, 1934, 43, 307.

[24] Titchener, A.L.; Bever, M.B.; Progress in Metal Physics. 1958, 7, 247.

[25] Bolshanina, M.A.; Panin, V.E.; Issledovaniya po Fisike Tverdogo Tela (Rus.). 1957, Collection of papers of the Tomsk State University (USSR) Tomsk,193.

[26] Salamatina, O.B.; Rudnev, S.N.; Paramzina, T.V.; Kravchenko, M.A.; Shantorovich, V.P.; Oleinik, E.F.; Polymer Sci. A. 2004, 46, 1815.

[27] Adams, G.W.; Farris, R.J.; Polymer, 1989, 60. 1829.

[28] Oleinik, E.F.; In: Relaxation in Polymers. 1980, Pietralla, M.; Pechhold, W.; Eds. Progress in Colloid Polym. Sci. Springer, Darmshtadt, 80,140.

[29] Godovskyi, Yu.K.; Thermophysical Properties of Polymers, 1993, Springer. Berlin. 
[30] Godovskyi, Yu.K.; Teplofisika polimerov (Rus.), 1982, Chemistry Publ. Moscow. [31] Obituary; Polym.Sci.; A 2005, 47, .

[32] Oleinik, E.F.; Salamatina, O.B.; Rudnev, S.N.; Shenogin S.V.; Polymers for Adv. Techn. 1993, 6, 1.

[33] Oleinik, E.F.; Adv. Pol. Sci. 1986, 80, 50.

[34] Nanzai, Y.; Konishi, T.; Ueda, S.; J.Mat.Sci. 1991, 26, 4477.

[35] Haward, R.; In: Physics of Glassy Polymers. 1973, Haward, R.N.;Ed., App;.Sci.Publ. London, 5.

[36] Fridel, J.; Dislocations. 1964, Pergamon Press, Oxford.

[37] Gilman, J.J.; in: Dislocation Dynamics, 1968, Rosenfeld, A.R.; et al. Eds. McGrew -Hill, N.Y. 3.

[38] Oleinik, E.; In: High Performance Polymers, 1990, Baer, E; Moet, E.; Eds. Hanser Verlag, Munich, 79.

[39] Oleinik, E.F.; Shenogin, S.; Paramsina, T.V.; Rudnev, S.N., Shantorovich, V.P., Azamatova, Z.K.; Pakula, T.; Fischer, E.W.; Polymer Sci. A. 1998, 40, 1944.

[40] Bershtein, V.A.; Egorov, V.M.; In:Differential Scanning Calorimetry in Polymer Physical Chemistry. 1990, Chemistry Publ., Leningrad, Ch.6.

[41] Salamatina, O.B.; Nazarenko,S.; Rudnev, S.N.; Oleinik, E.F.; Mechanics of Compos.Mater. (Rus.),1988, 6, 979.

[42] Salamatina, O.B.; Rudnev, S.N.; Oleinik, E.F.; J.Therm.Anal. 1992, 38, 1271.

[43] Quinson, R., Perez ,J., Rink, M., Pavan, A.; J.Mat.Sci. 1996, 31, 4387

[44] Kotelyanskyi, M.J.; Mazo, M.A.; Oleinik, E.F.; Grivtsov, A.G.; Phys.Stat.Sol. b. 1991, 166, 25

[45] Kotelyanskyi,M. J.; Ph.D.Thesis 1992, Institute of Chemical Physics, Moscow.

[46] Nelson, D.; Rubinshtein, M.; Phys.Rev.; B16, 1982 ,11, 6254. 